\title{
Automatic Modeling of an Orthotic Bracing for Nonoperative Correction of Pectus Carinatum
}

\author{
João L. Vilaça ${ }^{1,5}$, Pedro L. Rodrigues ${ }^{1,2}$, António H.J. Moreira ${ }^{1,2}$, João Gomes Fonseca ${ }^{1}$, A.C.M. \\ Pinho $^{3}$, Jaime C. Fonseca ${ }^{4}$, Nuno Rodrigues ${ }^{5,6}$ \\ ${ }^{1}$ ICVS/3B's - PT Government Associate Laboratory, Braga/Guimarães, Portugal \\ ${ }^{2}$ Algoritmi Center, School of Engineering, University of Minho, Guimarães, Portugal \\ ${ }^{3}$ Mechanical Department, University of Minho, Guimarães, Portugal \\ ${ }^{4}$ Industrial Electronics Department, University of Minho, Guimarães, Portugal \\ ${ }^{5}$ DIGARC - Polytechnic Institute of Cávado and Ave, Barcelos, Portugal \\ ${ }^{6}$ HASLab/INESC TEC, University of Minho, Braga, Portugal
}

Keywords: $\quad$ Pectus Carinatum, Nonoperative Treatment, 3D Image Processing, Mesh Processing

\begin{abstract}
Pectus Carinatum is a deformity of the chest wall, characterized by an anterior protrusion of the sternum, often corrected surgically due to cosmetic motivation. This work presents an alternative approach to the current open surgery option, proposing a novel technique based on a personalized orthosis. Two different processes for the orthosis' personalization are presented. One based on a 3D laser scan of the patient chest, followed by the reconstruction of the thoracic wall mesh using a radial basis function, and a second one, based on a computer tomography scan followed by a neighbouring cells algorithm. The axial position where the orthosis is to be located is automatically calculated using a Ray-Triangle intersection method, whose outcome is input to a pseudo Kochenek interpolating spline method to define the orthosis curvature. Results show that no significant differences exist between the patient chest physiognomy and the curvature angle and size of the orthosis, allowing a better cosmetic outcome and less initial discomfort.
\end{abstract}

\section{INTRODUCTION}

Pectus Carinatum is a deformity of the chest wall characterized by an anterior protrusion of the sternum and ribs. It occurs in approximately one out of every 1500 children (about $80 \%$ of patients are male). Current treatment for Pectus Carinatum includes both surgical and nonsurgical options. Despite the success of operative approaches, the related surgery complications (e.g. bleeding), scars and postoperative pain, present considerable drawbacks of these more invasive techniques (Alexander et al., 2009).

Based on the fact that the anterior chest wall is malleable during puberty, many authors advocate the benefits of nonoperative approaches to induce chest wall remodelling (Frey et al., 2006; Goretsky et al., 2004). Although this treatment option has been shown to provide positive results over time, some parents find it difficult to keep their children from taking off the brace (most of the times due to wearing discomfort), which reduces the overall therapeutic process efficiency (Stephenson and Du Bois, 2008).

The present work proposes two different approaches for the automatic orthosis personalization: one based on a 3D laser scan and another on a computer tomography (CT) scan of the chest.

Afterwards, the system developed in (Vilaça et al., 2009) is applied to automatically model and bend the orthosis bar, according to each patient morphology.

\section{METHODS AND RESULTS}

This section describes all methods to automatic determine the array points that are used in (Vilaça et al., 2009). 


\subsection{Thoracic Wall Reconstruction}

This stage reconstructs the anterior and posterior thoracic wall according to two different sets of input data, namely CT imagiology and 3D scanner dat.

Since CT scans remain the primary choice for preoperative imaging of Pectus Carinatum, a CT data set is available in most patients, providing useful information about abnormal thoracic anatomy and symmetry defects. Therefore, one has implemented an algorithm that automatically segments all skin points and generates a 3D mesh from a CT data set. The process starts with a preprocessing stage that performs a smooth operation on the CT data (using a Gaussian filter) and calculates its gradient volume magnitude. Then, all image pixels are vertically and horizontally tracked by recursively selecting all first voxels belonging to the image gradient (skin points). These voxels are input to a neighbouring cells algorithm, where each voxel of a cubic grid cell is classified as lying above or below an 800 iso-surface threshold. A tri-linear interpolation is then used to reconstruct a skin surface mesh from CT data (Figure 1-B).

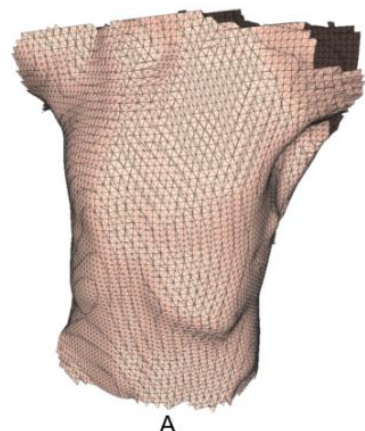

A

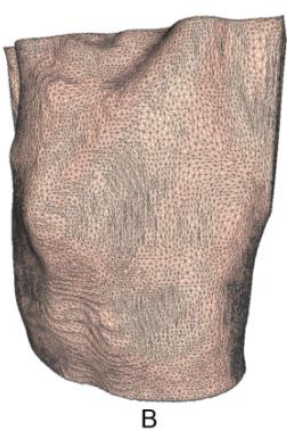

Figure 1: 3D Mesh representation from a 3D scan (A) and using a 3D CT data set.

Although CT scans have been the main analysis option to assess Pectus Carinatum severity, sometimes these are not available or are deprecated. For these patients, an inoffensive and radiation-free 3D laser scan using the FastSCAN system can be a much better choice, avoiding the need of a CT scan, and the related radiation, to reconstruct the thoracic wall. Exploring such an option, one was able to achieve a minimal error acquisition of approximately $1 \mathrm{~mm}$, by attaching the system reference to the $10^{\circ}$ left rib.

The unorganized point clouds acquired from the 3D scan, containing outlier's points, noise and nonuniformities in thickness and spacing were used to reconstruct the thoracic wall mesh surface. The algorithm used to reconstruct a smooth surface from the raw points of the 3D scanner was based on Radial Basis Functions (RBFs) (Savchenko et al., 1995).

The data misalignments and surface holes (when two or more samples overlap in the same Oxyz plane) were eliminated by modifying the voxel size and an adaptive kernel window during reconstruction. In the end, a $3 \mathrm{D}$ mesh was created representing the thoracic wall (Figure 1 - A).

After the mesh reconstruction, some surface holes can still exist. This situation was more frequent when the mesh was created using the $3 \mathrm{D}$ scanner, due to patient movements during the acquisition and light interference. To overcome this problem, a correction filter was developed, that searches for accentuated changes on the $\mathrm{X}$ and $\mathrm{Y}$ components of the discrete signal derivative. For each triangle $\mathrm{T}$ of the surface mesh whose partial derivative value is higher than a given threshold, when compared to its neighbourhood triangles (within a defined radius $\mathrm{R}$ ), the triangle vertices coordinates within $\mathrm{R}$ were merged together according to its neighbourhood maximum values. The outcome of this process was input to a Laplacian smooth method to reduce the shrinking effect.

\subsection{Highest sternal protrusion}

The surface mesh created on the previous section was input to a Ray-Triangle intersection method, which was used to compute the minimal distance between two triangle meshes: the thoracic wall mesh (Ts) and a plane mesh reference (Tp) which size was adjusted for the Ts size.

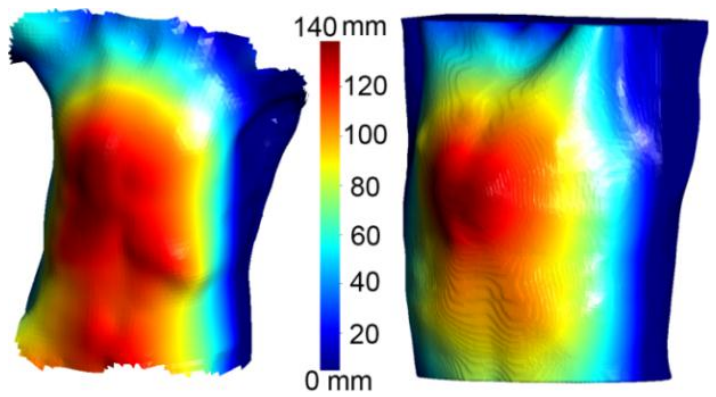

Figure 2: Illustration of the Ray-Triangle intersection method results, used to automatically determine the region of the greatest sternum protrusion.

In the end, the difference between each plane facet triangle and the thoracic facet triangle was determined. Based on these distances, the thoracic 
triangle facets were coloured according to the colour-map defined on the colour bar in Figure 2. The most anterior prominent sternal protrusion (red area, Figure 2), was chosen for orthosis placement and for the sizing of the cushioned compression plates of the anterior position.

\subsection{Orthosis automatic modelling}

The higher sternum protrusion point and a normal along Oy were firstly used to define an axial plane to place the orthosis. Then, a method was implemented to cut the thoracic wall mesh that intersects this plane.

The cutting result was a set of points that were input to a method that determines a pseudo Kochenek interpolating spline with a control continuity and tension parameter (Kochanek and Bartels, 1984).

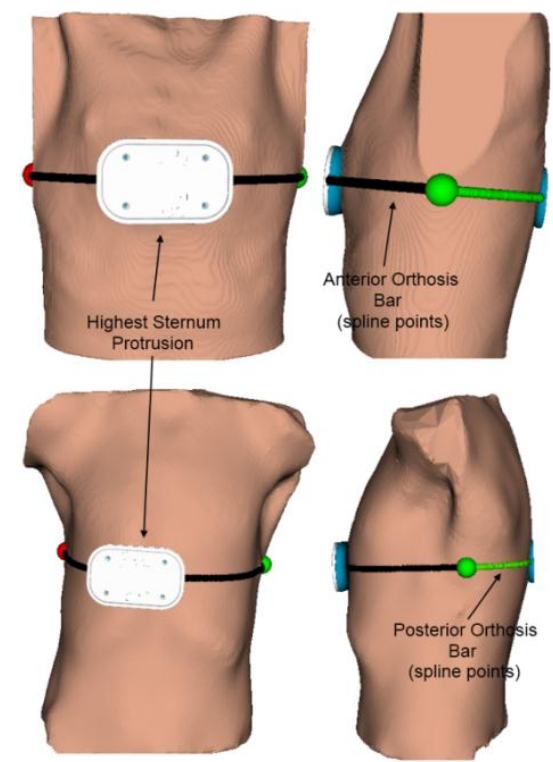

Figure 3: Bar modelling representation.

The continuity/discontinuity of the first derivative of at a key spline position was defined according to Equation 1:

$$
\begin{gathered}
\text { Out }_{\text {Spline }}=\left(\frac{1 \pm c}{2}\right) \cdot\left(P_{i}-P_{i-1}\right)+ \\
\left(\frac{1 \mp c}{2}\right)\left(P_{i+1}-P_{i}\right)
\end{gathered}
$$

where $\mathrm{c}$ is the continuity factor (experimentally calculated $-\mathrm{c}=0.2$.

This kind of spline produced fewer oscillations (when the thoracic mesh data is not smooth), had no overshoots, and preserved the data monotonicity. By using this spline it was possible to determine all regions where the prosthesis is placed, avoiding skin irregularities that are not relevant for the orthosis modelling.

The following methodology was then used to determine the final orthosis shape (Figure 3):

1) Determination of the rightist and leftist spline points which defines a coronal plane along the thoracic wall (red and green spheres, Figure 3). Each of these locations defines two planar regions (which size depends on the anterior-posterior patient length) on the anterior and posterior orthosis braces (LRA and LRP respectively in Figure 4), allowing (1) the placement of the linking area between the anterior and posterior orthosis braces; (2) the position of two mechanical systems for the physician to adjust the correction chest pressure to the desired level; and (3) the position of four cushioned compression plates to increase patient comfort;

2) Determination of the most anterior and posterior spline points. Those define the area where two cushioned plates are attached to the anterior and posterior segment of the brace (Figure 3 and Figure 4 (AC and $\mathrm{PC}$, respectively)). The size of each cushioned plates are also automatically determined with respect to the thoracic morphology and the deformity area (regions with higher red intensity, Figure 3).

3) Bar modelling, taking into account (1) the different positions determined at step 1 and 2; (2) an offset curvature by scaling each spline point along Oxyz normal, according to the cushioned plate's thicknesses (approximately $7 \mathrm{~mm}$ );

4) Finally, an array of points, containing the shape information of each posterior and anterior bars, is input to the (Vilaça et al., 2009) system to automatically model the entire orthosis bar (Figure 3 - spline points).

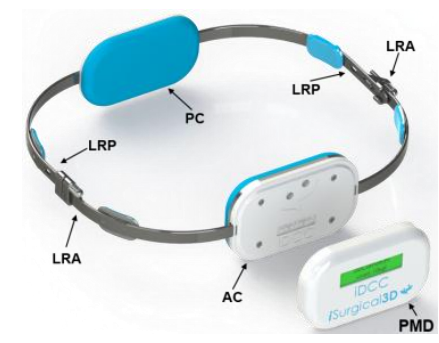

Figure 4: Final shape of the orthosis

\section{CONCLUSION}

Current orthosis are modelled and bended using control anatomical references that are identified manually. Such procedure is time consuming, leaves imperfections in the prosthesis surface and greatly 
depends on the physician's experience. Moreover, most of these orthosis are modelled independently of the symmetric defect and do not take into consideration the thoracic wall shape. Consequently, this process often produces non-uniform strength distributions, misalignments and offsets between the orthosis and the patient thoracic wall (which end up producing initial discomfort), thoracic pain, increased patient adaptation time and decreased cosmetic outcome of the deformity. To overcome current orthosis practice disadvantages, this work proposes a systematic methodology for the personalisation of a Pectus Carinatum orthosis. The orthosis size and curvature are automatically determined using information retrieved by CT imagiology or a $3 \mathrm{D}$ scanner of the thoracic wall shape.

An array of points containing this virtual model information is input to the (Vilaça et al., 2009) system to automatically bend two stainless steel braces (AISI type 316LVM (low carbon vacuum melt), corresponding to the anterior and posterior orthosis braces. The differences between the virtual and physical braces were determined by modelling and bending 15 orthosis using $15 \mathrm{CT}$ data sets of patients with Pectus Carinatum, acquired at São João Hospital of Porto (Portugal). To measure these differences, an LVTD (linear variable differential transformer) was used to compare, at different bend times, the differences between the virtual point and curvature, with the real values. Results show that no significant differences exist concerning the curvature angle and size of the orthosis, given that all errors are below $10 \mu \mathrm{m}$.

Recently, this personalized orthosis has been modelled for two patients whose thoracic wall showed symmetric (Figure 1 - B) and asymmetric defects (Figure 1 - A). For both patients, the orthosis suitably fitted the thoracic wall shape, and the pressure of the cushioned compression plates was adequate to prevent slippage. The pressure of treatment is controlled using an electronic pressure sensor whose output value is shown on an LCD (PMD in Figure 4). This permits to adjust the correction pressure to the desired level and prevents from making too much pressure, which could cause pressure necroses.

Such results indicate a considerable step forward that might decrease the need of open surgery for a nonoperative approach in Pectus Carinatum deformity correction. In addition, nonoperative management offers a significant cost benefit since with this new method, hospitalization time, per- operative and post-surgical complications are eliminated.

\section{ACKNOWLEDGEMENTS}

The authors acknowledge to Foundation for Science and Technology (FCT) - Portugal for the fellowships with the references: SFRH/BD/74276/2010; SFRH/BD/68270/2010; UMINHO/BI/95/2012; and, $\mathrm{SFRH} / \mathrm{BPD} / 46851 / 2008$. This work was also supported by FCT R\&D project PTDC/SAUBEB/103368/2008.

\section{REFERENCES}

Alexander, A.F., Nury, M.S., William, A.A., Karen, E.A., 2009. Anatomical, Histologic, and Genetic Characteristics of Congenital Chest Wall Deformities. Seminars in thoracic and cardiovascular surgery 21 , 44-57.

Carr, J.C., Beatson, R.K., McCallum, B.C., Fright, W.R., McLennan, T.J., Mitchell, T.J., 2003. Smooth surface reconstruction from noisy range data, Proceedings of the 1st international conference on Computer graphics and interactive techniques in Australasia and South East Asia. ACM, Melbourne, Australia, pp. 119-ff.

Frey, A.S., Garcia, V.F., Brown, R.L., Inge, T.H., Ryckman, F.C., Cohen, A.P., Durrett, G., Azizkhan, R.G., 2006. Nonoperative management of pectus carinatum. J Pediatr Surg 41, 40-45; discussion 40-45.

Fritsch, F.N., Carlson, R.E., 1980. Monotone Piecewise Cubic Interpolation. SIAM Journal on Numerical Analysis 17, 238-246.

Goretsky, M.J., Kelly, R.E., Jr., Croitoru, D., Nuss, D., 2004. Chest wall anomalies: pectus excavatum and pectus carinatum. Adolesc Med Clin 15, 455-471.

Kochanek, D.H.U., Bartels, R.H., 1984. Interpolating splines with local tension, continuity, and bias control. SIGGRAPH Comput. Graph. 18, 33-41.

Savchenko, V.V., Pasko, A.A., Okunev, O.G., Kunii, T.L., 1995. Function Representation of Solids Reconstructed from Scattered Surface Points and Contours. Comput Graph Forum 14, 181-188.

Stephenson, J.T., Du Bois, J., 2008. Compressive orthotic bracing in the treatment of pectus carinatum: the use of radiographic markers to predict success. Journal of pediatric surgery 43, 1776-1780.

Vilaça, J., Pinho, A., Correia-Pinto, J., Fonseca, J., Peixinho, N., 2009. System for automatic and personalized modelling/bending of surgical prosthesis for correction of pectus excavatum based on presurgical imagiology information, WO2009/035358. 